\title{
Ansiedade e sua relação com a qualidade de vida em idosos: revisão narrativa
}

\author{
Anxiety and its relationship with quality of life \\ In elderly: narrative review
}

Daniel Vicentini de Oliveira, ${ }^{1}$ Mateus Dias Antunes, ${ }^{2}$ Julimar Fernandes de Oliveira'

${ }^{1}$ Universidade Estadual de Campinas (Unicamp), Campinas, SP, Brasil.

2 Centro Universitário de Maringá (Unicesumar) Maringá, PR, Brasil.

Recebido em: 13/07/2017 / Aceito em: 10/09/2017 / Publicado em: 01/10/2017

d.vicentini@hotmail.com

\section{RESUMO}

Objetivo: identificar a relação entre ansiedade e qualidade de vida dos idosos. Método: foi realizada uma revisão narrativa, de artigos publicados entre 2012 a 2016, em periódicos nacionais e internacionais indexados nas bases de dados Scielo, Lilacs e Medline. Os descritores utilizados foram: ansiedade, qualidade de vida e idosos no idioma português, espanhol e inglês. Buscaram-se artigos que estavam disponíveis na íntegra e que apresentavam as palavras chaves qualidade de vida, ansiedade e idoso. Resultados: a ansiedade é definida como um sentimento desagradável, difuso e vago; pode afetar os idosos na sua percepção de qualidade de vida. Estudos demonstram que há uma relação inversamente proporcional entre a qualidade de vida, com os níveis de ansiedade, ou seja, há um impacto negativo dos transtornos de ansiedade sobre a qualidade de vida. Considerações finais: as manifestações psicológicas inadequadas em termos de intensidade, frequência ou duração, como a ansiedade, podem trazer desconfortos no bem-estar e consequentemente interferir na qualidade de vida, primordialmente pela restrição de sua vida social.

Palavras-chave: Transtornos de ansiedade; Qualidade de Vida; Promoção da Saúde.

\section{ABSTRACT}

Objective: to identify the relationship between anxiety and quality of life of the elderly. Method: a narrative review of scientific articles published between 2012 and 2016 was carried out in national and international journals indexed in the Scielo, Lilacs and Medline databases. The descriptors used were: anxiety, quality of life and elderly in Portuguese, Spanish and English. We searched for articles that were available in full and which featured the key words quality of life, anxiety and seniority. Results: anxiety is defined as an unpleasant, diffuse and vague feeling which can affect the elderly in their perception of quality of life. Studies have shown that there is an inversely proportional relationship between quality of life and anxiety levels, that is, there is a negative impact of anxiety disorders on quality of life. Closing remarks: Inadequate psychological manifestations in terms of intensity, frequency or duration such as anxiety can bring discomfort to well-being and consequently interfere with quality of life, primarily by restricting social life of elderly.

Keywords: Aging; Anxiety Disorders; Quality of Life; Health Promotion. 


\section{INTRODUÇÃO}

O processo de senescência é considerado dinâmico, gradativo e repleto de mudanças físicas e psicossociais. Nesse sentido, as mudanças tornam os idosos mais vulneráveis e susceptíveis a determinados agravos na saúde, bem como o surgimento de doenças. ${ }^{1}$ Com isso, essas perdas provocam sentimentos de ansiedade, medo, tristeza, irritação e a necessidade de adaptação de um novo estilo de vida. ${ }^{2}$

A prevalência de patologias associadas aos idosos, aumenta os sintomas de ansiedade e depressão, sendo consideradas como relevantes problemas de Saúde Pública. ${ }^{3}$ Algumas patologias são fontes de sofrimento emocional e consequentemente reduzem a qualidade de vida dos idosos. ${ }^{4}$

Com o avanço da idade e o aumento das perdas fisiológicas, às vezes podem estar acompanhadas por perdas físicas, sociais, emocionais ou cognitivas; a ansiedade surge atuando na redução da capacidade cognitiva, perda de memória e aumentando os riscos de doencas. ${ }^{5} \mathrm{Na}$ terceira idade, a ansiedade pode estar ligada também à insuficiência de suporte físico social e dificuldades nas relações interpessoais, levando a sofrimento psíquico. ${ }^{4}$

A ansiedade é considerada uma reação natural até certo ponto, útil para proteção e adaptação a novas situações, mas torna-se patológica, quando atinge um caráter extremo e generalizado, acompanhado por sintomas de tensão, medo, em que o foco do perigo pode ser externo ou interno. A mesma tem um impacto negativo sobre a qualidade de vida dos idosos, ${ }^{6}$ portanto, faz-se necessário o estudo dessas variáveis. Diante do exposto, o presente estudo teve por objetivo identificar a relação existente entre ansiedade e suas consequências para a qualidade de vida do idoso.

\section{MÉTODO}

Para o desenvolvimento do presente estudo foi realizada uma revisão narrativa que analisou artigos publicados entre 2012 e 2016, nos periódicos nacionais e internacionais indexados nas bases de dados Scielo, Lilacs e Medline. Os descritores utilizados foram: ansiedade, qualidade de vida e idosos no idioma português, espanhol e inglês. Buscou-se artigos que estavam disponíveis na íntegra.

O período de busca foi entre os meses de Novembro a Dezembro de 2016 e após selecionar os artigos para extrair as definições sobre o tema os autores procederam à leitura dos mesmos. Foram excluídos teses, dissertações e resumos publicados em anais.

\section{REVISÃO DE LITERATURA}

\section{O PROCESSO DE ENVELHECIMENTO}

Tem mudado rapidamente nos últimos anos o perfil epidemiológico e demográfico da população brasileira. Ao contrário da transição demográfica, que ocorreu lentamente e acompanhando a elevação da qualidade de vida nos países desenvolvidos, no Brasil essa mudança está acontecendo de forma rápida. ${ }^{7}$
O envelhecimento populacional é considerado um fenômeno comum na maioria dos países do mundo. No entanto, o aumento na proporção de idosos e redução das crianças não ocorre igual em todos os lugares. Enquanto nos países desenvolvidos o enveIhecimento é gradual, nos países em desenvolvimento é acelerado e está acontecendo antes do almejado desenvolvimento econômico. ${ }^{8}$

Este aumento da expectativa de vida da população mundial faz com que os idosos tornem-se tema principal em discussões, a fim de se alcançar um envelhecimento com mais saúde e menor dependência. ${ }^{9}$

A inversão da pirâmide populacional é decorrente de diversos fatores entre os quais, a redução das taxas de fecundidade, fertilidade, natalidade e mortalidade infantil, a melhoria nas condições de infraestrutura e saneamento básico, o aumento da expectativa de vida, tem demonstrado um elevado aumento da longevidade da população. ${ }^{10}$

A expectativa de vida tem aumentado no Brasil, inclusive na faixa etária de 80 anos ou mais e, principalmente, para o sexo feminino, tornando-se um preocupante fato, especialmente nos aspectos relacionados à qualidade de vida e à saúde deste grupo etário. ${ }^{11}$ No Brasil, a proporção dos idosos passou de 9,7\% em 2004 para $13,7 \%$ em $2014 . .^{12}$ Essa tendência vai continuar e em 2060 chegará em 33,7\%, ou seja, a cada três pessoas, uma vai ser idosa. ${ }^{13}$

Alguns autores também justificam o aumento da expectativa de vida, como sendo o reflexo de mudanças culturais, avanços nas tecnologias em saúde e melhorias nas condições de vida da população. ${ }^{14}$ Além desses fatores, a melhoria do acesso à saúde pela população, as campanhas de vacinação, prevenção e controle de doenças, porém atrelado a esses fatores. Houve uma transição epidemiológica, em que as doenças infecciosas foram substituídas por doenças crônicas-degenerativas, como causa de morbimortalidade. ${ }^{15}$

Linearmente, a expectativa de vida vem aumentando, bem como as desabilidades e as doenças, as quais têm direta influência na qualidade de vida e autonomia dos idosos. ${ }^{16}$ Com o aumento dessa expectativa de vida, objetiva-se um envelhecimento saudável e bem sucedido e com qualidade de vida. ${ }^{17}$

O envelhecimento é caracterizado como um processo dinâmico e progressivo, em que ocorrem transformações importantes nos aspectos morfofuncionais, biofisiológicos e psicossociais. Neste período, as perdas são graduais, ocorrem em ritmo diferenciado e individual e sofrem interferência de diversos fatores. ${ }^{18}$ Ao longo da vida, o organismo sofre diversas alterações causadas por fatores intrínsecos e extrínsecos que aceleram o processo de envelhecimento, o que gera modificações fisiológicas inevitáveis e irreversíveis. ${ }^{19}$

O envelhecimento é um processo natural dos seres vivos. Algumas mudanças celulares e fisiológicas, que se apresentam com a longevidade, são reflexos das enfermidades, estilo de vida, cultura, sexo, educação e condição socioeconômica. ${ }^{20}$ Os fatores que impulsionam o envelhecimento populacional são distintos de outras experiências, em parte, pela heterogeneidade econômica, social, cultural e ambiental. ${ }^{21,22}$

Junto com o envelhecimento, nota-se o aumento 
das doenças crônico-degenerativas e suas sequelas, bem como a procura por serviços de saúde. ${ }^{23,24} \mathrm{~A}$ longevidade tem sido acompanhada por diversas alterações sociais e familiares. ${ }^{25}$ Com o avanço da idade ocorrem declínios fisiológicos cumulativos nos diversos sistemas corporais, caracterizados por alterações estruturais e funcionais. ${ }^{26}$ Essas mudanças comprometem o desempenho de habilidades motoras que interferem negativamente na qualidade de vida. ${ }^{27}$

Neste contexto, como consequência de uma população envelhecida, a educação e a promoção em saúde, à prevenção e retardamento das fragilidades e das doenças, a manutenção da autonomia e independência, são iniciativas que devem ser ampliadas no âmbito mundial. ${ }^{28}$

\section{ALTERAÇÕES PSICOLÓGICAS DO ENVELHECIMENTO}

Já é sabido que o processo de envelhecimento envolve entre outras coisas, um declínio cognitivo que é fisiológico, que se dá em detrimento do processo de degeneração do sistema nervoso, o qual inclui várias alterações estruturais. ${ }^{29} \mathrm{O}$ sistema nervoso central é incapaz de realizar reparos nas alterações morfológicas adquiridas com a senescência. ${ }^{30} \mathrm{~A}$ partir da segunda década de vida, é evidenciado o envelhecimento cerebral, nota-se um declínio discreto, lento e progressivo, que culmina com a redução do seu volume. ${ }^{31}$

O termo cognição corresponde à faixa de funcionamento intelectual humano, incluindo atenção, percepção, raciocínio, memória, tomada de decisões, solução de problemas e formação de estruturas complexas do conhecimento. ${ }^{32}$ A dificuldade maior acerca do envelhecimento é o limite entre alterações normais cognitivas e patogênicas. No entanto, o conhecimento dos diversos tipos de demências, o avanço dos métodos de avaliação e as evidências científicas permitiram o julgamento sobre o limite entre saúde e doença no idoso. ${ }^{33}$

Em relação ao tempo, algumas habilidades cognitivas se modificam, enquanto outras permanecem inalteradas. As habilidades que sofrem declínio com a idade são: memória de trabalho, velocidade de habilidades visoespaciais e de pensamento, enquanto as que se mantêm inalteradas são: inteligência verbal, habilidade de cálculo, atenção básica e a maioria das habilidades de linguagem. ${ }^{34}$

As regiões que estão mais sensíveis às alterações do envelhecimento localizam-se no lobo frontal e, possivelmente, no lobo temporal médio. Além disso, alterações dos órgãos dos sentidos como, visão, audição, tato e olfato dificultam o acesso às informações e o aprendizado. ${ }^{31}$

A capacidade intelectual do idoso pode ser mantida sem dano cerebral até os 80 anos. No entanto, o declínio cognitivo com o envelhecimento pode variar quanto ai início e progressão, pois alguns fatores são dependentes; como educação, personalidade, saúde, nível intelectual global, capacidade mental específica, entre outros. ${ }^{35}$ Alguns fatores influenciam o envelhecimento cognitivo, sendo eles genéticos, estado de saúde, instrução, atividade mental, conhecimento especializado, personalidade, humor, treino cognitivo e o meio social e cultural. ${ }^{36}$

O envelhecimento pode levar ao declínio no de- sempenho cognitivo, como a redução da atenção e da velocidade do processamento de informação. ${ }^{37} \mathrm{As}$ mudanças psicológicas com o envelhecimento resultam da dificuldade de adaptações a novos papéis sociais, falta de motivação, baixa-estima, dificuldade de mudanças rápidas, perdas afetivas e orgânicas, somatização, hipocondria e depressão. ${ }^{30}$

A ansiedade e a depressão são os agravos psiquiátricos mais comuns na velhice e estão associadas à percepção de suporte social como negativo ou insuficiente, ao menor grau de proximidade com outras pessoas e à menor satisfação com as relações sociais. ${ }^{38} \mathrm{~A}$ presença de ansiedade e depressão nos idosos está associada ao sofrimento psíquico e físico, decorrente do agravamento de incapacidades e doenças crônicas, às perdas de entes queridos e papéis sociais e ao histórico prévio de transtornos psiquiátrico. ${ }^{39} \mathrm{~A}$ idade avançada é um dos fatores que contribuem para um maior desenvolvimento de sintomas depressivos. ${ }^{40}$

A manutenção dos aspectos psicológicos auxilia na promoção da independência e autonomia do idoso e a sua estimulação a fim de prevenir ou até mesmo retardar o processo de degeneração do cérebro ${ }^{41}$. Os idosos que ao longo da vida permanecem ativos apresentam inúmeros ganhos para a saúde, beneficiando-se com melhoras no campo do bem-estar psicológico e da qualidade de vida. ${ }^{36}$

\section{ANSIEDADE EM IDOSOS}

Para elaborar novos significados na velhice, deve-se incentivar, fundamentalmente, a prevenção, o cuidado e a atenção integral à saúde, seja física ou metal. Logo se faz necessário compreender que na saúde mental há diversos transtornos psicológicos, entre eles variados níveis de ansiedade, situação que é altamente prevalente entre os idosos. ${ }^{42,43}$

A ansiedade é algo comum faz parte da vida das pessoas, pode ser um sentimento útil, servido de sinal de alerta, diante de circunstancias conflitantes, entretanto quando os níveis de ansiedade estão acima do controlável, pode desencadear sentimentos desagradáveis como palpitações, dor no peito, desconforto abdominal, inquietação, situações que interferem na saúde física e mental da pessoa acometida e primordialmente os idosos, ao estarem fragilizados pela influencia da idade, tendem a ampliar tais sintomas prejudicando a qualidade de vida dos mesmos. ${ }^{42-45}$

Há que se destacar a maior prevalência de ansiedade em idosos, com menor grau de escolaridade ou que apresentam doenças crônicas. A ansiedade é mais comum, também, em mulheres ao se comparar com homens, e os sintomas mais acentuados, surgem primordialmente no começo da vida adulta. Os danos da ansiedade na saúde se destacam por restringir os idosos da vida social, diminuir a independência e naturalmente afetar negativamente a qualidade de vida. ${ }^{45}$

A ansiedade é considerada a nona causa de incapacidade no mundo; ${ }^{46}$ os seus principais sintomas estão associados a doenças psiquiátricas, principalmente a depressão, causando um impacto importante na qualidade de vida e, quando associados à dependência física, típica de pessoas com 60 anos ou mais, intensificam as limitações para atividade de vida diária 
(AVD) e incapacitam os idosos a uma vida cotidiana. Há evidencias que as doenças mais prevalentes nos idosos como as cardiovasculares, hipertensão arterial sistêmica e infarto agudo do miocárdio (IAM) ou as doenças respiratórias podem agravar a ansiedade ou essa ser agravada por tais doenças. ${ }^{47}$

A ansiedade está envolta de distúrbios do humor, com manifestações afetivas negativas ou inadequadas em termos de intensidade, frequência e duração. Nos idosos está associada à depressão, fragilidade, desnutrição, baixa autoestima, gerando um sofrimento psicológico. ${ }^{48}$ Logo, a ansiedade pode causar maior prevalência de doença física, comprometimento psicossocial e mortalidade. Afeta milhões de pessoas independentemente de etnia, educação, sexo ou renda, e possui forte prevalência entre os idosos. ${ }^{49} \mathrm{~A}$ ansiedade pode, ainda, prejudicar a qualidade de vida, aumentar os riscos de sofrimento e incapacidade. ${ }^{45-50}$

Os idosos com ansiedade tendem a possuir níveis significativamente mais elevados de aflição, comprometimento funcional, piora na qualidade de vida e mais busca por consultas de saúde, e possivelmente, traz maior custos aos sistemas de atendimento em saúde. ${ }^{51}$ Idosos que apresentam queixas de memória, tendem a apresentar, mais sentimentos de ansiedade e de estresse, sendo mediada por alterações psicológicas.

Nos estudos sobre ansiedade, há diversos modelos de escalas para rastreio dos diferentes níveis e sintomas, um dos mais aplicados na literatura e na prática clínica é o Inventário de Ansiedade de Beck (BAI), o qual foi validado no Brasil por Cunha. ${ }^{52}$ Esse é composto pode uma lista com 21 sintomas mais comuns da ansiedade; aborda questões relacionadas à saúde, próprias do envelhecimento, problemas financeiros e medo de abandono que podem gerar ansiedade no idoso, e auxiliam na compreensão das situações que podem repercutir na percepção negativa ou positiva da qualidade de vida. ${ }^{53}$

\section{QUALIDADE DE VIDA DO IDOSO}

A saúde da população idosa brasileira possui aspectos característicos da idade que pode gerar reflexos na qualidade de vida que é afetada pelas características do contexto social, dos distúrbios psicológicos das doenças crônicas e do estilo de vida. É sabido, que para determinar a qualidade de vida do idoso é necessário sistematizar seus determinantes ou situações impactantes, como a ansiedade. ${ }^{33}$

A qualidade de vida (OV) é, também, em sua essência central à aptidão, em viver sem doenças ou as superar com maior velocidade, quando aparecem. É ainda, uma noção eminentemente humana, que aproxima ao grau de satisfação encontrado na vida. O termo contem muitas definições, que conjecturam conhecimentos, vivencias e valores individuais dentro da comunidade e que se mesclam com as várias épocas e com o contexto histórico vivenciado. Abrange variados aspectos como o nível socioeconômico e o estado emocional, os valores culturais, éticos e a religiosidade ou o próprio o estilo de vida. ${ }^{33,54}$

Como o significado de qualidade de vida é extremamente abrangente, entende-se ser necessário avaliá-la e compreendê-la como um importante indicador de saúde, já que apresenta características relacionadas às condições de bem-estar físico, mental e social. Além disso, analisá-la no contexto psicológico, pode influenciar em novos entendimentos de sua relação com a saúde mental, para que assim direcionem estratégias de cuidado para a melhoria da vida das pessoas, em especial do idosos. ${ }^{55}$

Pesquisas acerca da qualidade de vida implicam em compreender a saúde. Assim, é necessário entender sobre os transtornos psicológicos que ameaçam a saúde mental, como a ansiedade, e para isso buscar a prevenção e alívio do sofrimento, como uma identificação precoce, avaliação e tratamento dos idosos com problemas de natureza psicológicas. ${ }^{56}$

Faz-se aqui necessário, compreender que a percepção da QV é vivenciada de forma individual e especifica de cada pessoa. A Organização Mundial da Saúde (OMS) define-a como à "percepção do indivíduo de sua posição na vida, no contexto da cultura e sistema de valores nos quais ele vive e em relação aos seus objetivos, expectativas, padrões e preocupações". ${ }^{33}$

\section{ANSIEDADE E QUALIDADE DE VIDA EM IDOSOS}

A qualidade de vida, portanto, pode sofrer influência de diferentes circunstâncias, gerando diferentes níveis de ansiedade. ${ }^{56} \mathrm{~A}$ ansiedade é definida como um sentimento desagradável, difuso e vago; pode afetar os idosos na sua percepção de qualidade de vida. Há necessidade crescente de se compreender como se molda as influencias da ansiedade sobre a saúde do idosos. ${ }^{57}$ Fazzi e col. ${ }^{57}$ demonstra que há uma relação inversamente proporcional entre a qualidade de vida com a ansiedade, ou seja, há um impacto negativo dos transtornos de ansiedade, sobre a qualidade de vida em idosos com indicação por exemplo, de cirurgia de catarata. As manifestações psicológicas inadequadas em termos de intensidade, frequência ou duração, como a ansiedade, podem trazer desconfortos no bem-estar e consequentemente interferir na qualidade de vida, primordialmente pela restrição de sua vida social. ${ }^{42}$

Houve um envelhecimento populacional importante nos últimos 50 anos, com o prolongamento do tempo de vida e uma diminuição das taxas de natalidade. As pessoas mais velhas procuram não só uma maior duração da vida, mas também uma melhor qualidade de vida. ${ }^{58} \mathrm{O}$ conceito de "qualidade de vida" é definido como uma percepção de satisfação e satisfação global em vários domínios-chave, com ênfase especial no bem-estar. A qualidade de vida possui um conceito subjetivo, dependente do nível sociocultural, da faixa etária e das aspirações pessoais do indivíduo e pode representar felicidade e prazer em variados aspectos da vida, situações que podem ser comprometidas pela ansiedade. ${ }^{33}$

Alguns estudos admitem que os padrões de saúde, em diferentes domínios da qualidade de vida, são piores entre os idosos com altos níveis de ansiedade. Além de afirmarem que há piores escores de QV, nos campos da vitalidade e nos aspectos sociais, com retraimento social. ${ }^{59} \mathrm{~A}$ ansiedade está intimamente associada com o aumento da mortalidade e utilização de cuidados de saúde e têm um impacto sobre a qualidade de vida dos idosos. A ansiedade é ainda, um preditor para maior fragilidade e aumento no número de internações. ${ }^{42}$ 
Os transtornos mentais relacionados à saúde têm sido associados à piora na percepção de $\mathrm{QV}$, por parte dos idosos. É crucial elaborar estudos que auxiliem na compreensão de como ocorrem tais influencias para melhorar a QV para os idosos, pois a saúde do idoso deve ser avaliada, tanto na sua condição física, como mental. Considerando todo esse contexto nota-se que a ansiedade sofre impacto dos problemas cognitivos próprios dos idosos e isso traz piora sobre a QV.43

\section{CONSIDERACִÕES FINAIS}

Vários estudos tendem a demonstrar que o apoio psicológico e com atividades de intervenção de grupos educativos parece ter uma influência favorável na redução dos sintomas de ansiedade e na melhor percepção de qualidade de vida. Além disso, certo nível mínimo de atividade física traz efeitos positivos em ambas circunstâncias. Logo, o controle da ansiedade pode, na maioria das vezes, melhorar a saúde mental, facilitar e fomentar o contato social e, influenciar favoravelmente a qualidade de vida, levando a uma melhor vida social e bem-estar geral no idoso. Há necessidade de estudos populacionais, com objetivos de entender melhor e investigar os mecanismos biopsicossociais envolvidos na relação entre ansiedade e a qualidade de vida, que permitam melhor compreender a população idosa.

\section{REFERÊNCIAS}

1. Wamser EL, Valderramas SR, Paula JÁ, Schieferdeckerd MEM, Amatante TP, Coelho RA, Stanczk L, Guimarães ATB, Gomes ARS. Melhor desempenho no teste timed up and go está associado a melhor desempenho funcional em idosas da comunidade. Geriatrics, Gerontology and Aging 2015;9(4):138-43. doi: 10.5327/Z24472115201500040003

2. Santos CA, Ribeiro AQ, Rosa COR, Ribeiro RCL. Depressão, déficit cognitivo e fatores associados à desnutrição em idosos com câncer. Ciênc Saúde Coletiva 2015;20(3):75160. doi: 10.1590/1413-81232015203.06252014

3. Tavares KO, Scalco JC, Vieira L, Silva JR, Bastos CCCB. Envelhecer, adoecer e tornar-se dependente: a visão do idoso. Kairós Gerontologia 2013;15(2):105-18.

4. Gomes JB, Reis LA. Descrição dos sintomas de Ansiedade e Depressão em idosos institucionalizados no interior da Bahia, Brasil. Kairós Gerontologia 2016;19(1):175-91.

5. Nordon DG, Guimarães RR, Kozonoe DY, Mancilha VS, Dias Neto VS. Perda cognitiva em idosos. Revista da Faculdade de Ciências Médicas de Sorocaba 2009;11(3):5-8.

6. Cheik NC, Reis IT, Heredia RA, Ventura ML, Tufik S, Antunes HK, Mello MT. Efeitos do exercício físico e da atividade física na depressão e ansiedade em indivíduos idosos. Revista Brasileira de Ciência e Movimento 2008;11(3):45-52.

7. Vargas LS, Lara MVS, Carpes PBM. Influência da diabetes e a prática de exercício físico e atividades cognitivas e recreativas sobre a função cognitiva e emotividade em grupos de terceira idade. Rev Bras Geriatr Gerontol 2014;17(4):867-78. doi: 10.1590/1809-9823.2014.13178

8. Mari FR, Alves GG, Aerts DRGC, Camara S. O processo de envelhecimento e a saúde: o que pensam as pessoas de meia-idade sobre o tema. Rev Bras Geriatr Gerontol
2016;19(1):35-44. doi: 10.1590/1809-9823.2016.14122

9. Carvalho $E$, Arantes RC, Cintra ASR. A inserção de idosos do Instituto Henrique da Silva Semente (IHESS) no município de Indaiatuba/SP na era digital: contribuições fisiogerontológicas. Rev Bras Geriatr Gerontol 2016;19(4):56775. doi: 10.1590/1809-98232016019.150036

10. Vilela $A B$, Carvalho PAL, Araújo RT. Envelhecimento bemsucedido: representação de idosos. Saúde 2016;2(2):101-14.

11. Silva JLS, Guedes RML. Efeitos de um Programa de Ginástica Orientada sobre os Níveis de Flexibilidade de Idosos. Saúde e Pesquisa 2015;8(3):541-8. doi: 10.17765/1983-1870.2015v8 n3p541-548

12. Carmo JF, Oliveira ERA, Morelato RL. Incapacidade funcional e fatores associados em idosos após o Acidente Vascular Cerebral em Vitória - ES, Brasil. Rev Bras Geriatr Gerontol 2016;19(5):809-18. doi: 10.1590/180998232016019.150215

13. Instituto Brasileiro de Geografia e Estatística. Síntese de indicadores sociais: uma análise das condições de vida da população brasileira. Rio de Janeiro: IBGE; 2015.

14. KuchemannBA. Astrid. Envelhecimento populacional, cuidado e cidadania: velhos dilemas e novos desafios. Sociedade e Estado 2012;27(1):165-80. doi: 10.1590/S010269922012000100010

15. Paula CLM, Santos EVL, Maia CGGS, Gouveia Filho OS, Sousa MNA. Qualidade de vida de idosos participantes de um grupo de convivência no munícipio de São MamedePB. REBES 2016;6(2):1-7. doi: 10.18378/rebes.v6i2.4018

16. Baliza GA, Lopes RA, Dias RC. O papel da catastrofização da dor no prognóstico e tratamento de idosos com osteoartrite de joelho: uma revisão crítica da literatura. Rev Bras Geriatr Gerontol 2014;17(2):439-49. doi: 10.1590/S180998232014000200020

17. Melo Neto JS, Marques AEZS, Gomes FC. Profile of Pneumopathic Elderly Persons Admitted to a Pulmonary Rehabilitation Center. Rev Bras Geriatr Gerontol 2016.19(5):759-67. doi: 10.1590/180998232016019.150143

18. Ribeiro CG, Shimosaka AM, Costa D. Qualidade de vida dos servidores em processo de envelhecimento-IFPR Campus Palmas. Revista FisiSenectus 2016;3(2):13-23. doi: 10.22298/rfs.2015.v3.n2.3180

19. Neman FA, Silva NH. O perfil de saúde dos indivíduos idosos residentes no município de Guarulhos São Paulo. Science in Health 2011;2(2):83-92.

20. Barbon FJ, Weutholter P, Flores RA. Alterações celulares no envelhecimento humano. Journal of Oral Investigations 2016;5(1):61-5. doi: 10.18256/2238-510X/j. oralinvestigations.v5n1p61-65

21. Berlezi EM, Farias AM, Dallazen F, Oliveira KR, Pillatt AP, Fortes CK. Como está a capacidade funcional de idosos residentes em comunidades com taxa de envelhecimento populacional acelerado?. Revista Brasileira de Geriatria e Gerontologia 2016;19(4):643-52. doi: 10.1590/180998232016019.150156

22. Campos ACV, Almenida MHM, Campos GV, Bogutchi TF. Prevalência de incapacidade funcional por gênero em idosos brasileiros: uma revisão sistemática com metanálise. Rev Bras Geriatr Gerontol 2016;19(3):545-59. doi: 10.1590/180998232016019.150086

23. Araújo APS; Mincoff RCL, Blanco PHM, Lordani TVA, Machado ES, Silva PDN, Oliveira DV, Yamaguchi UM. Análise da Evolução Temporal da Morbidade Hospitalar 
de Idosos na Região da Amusep entre os anos de 2000-2010. Saúde e Pesquisa 2015;8(2):307-16. doi: 10.17765/1983-1870.2015v8n2p307-316

24. Becker AC, Falcão DVS. O envelhecimento, a velhice e o significado de ser avô (ó) na perspectiva de atores profissionais idosos. Rev Bras Geriatr Gerontol 2016;19(2):289-302. doi: 10.1590/1809-98232016019.140212.

25. Carvaho MS, Martins JCA. O Cuidado Paliativo a Idosos Institucionalizados: Vivência dos Ajudantes de Ação Direta. Rev Bras Geriatr Gerontol 2016;19(4):745-58. doi: 10.1590/1809-98232016019.150178

26. Hartmann Junior JAS, Gomes GC. Depressão em idosos institucionalizados: padrões cognitivos e qualidade de vida. Ciências \& Cognição 2016;21(1):137-54.

27. Gomes GC, Samela LFT, Freitas AS, Fonseca MLM, Pinheiro MB, Morais VAC, Caramello P. Desempenho de idosos na marcha com dupla tarefa: uma revisão dos instrumentos e parâmetros cinemáticos utilizados para análise. Rev Bras Geriatr Gerontol 2016;19(1):165-82. doi: 10.1590/18099823.2016.14159

28. Muniz EA, Aguiar MFS, Brito MCC, Freitas CASL, Moreira ACA, Araújo CRC. Desempenho nas atividades básicas da vida diária de idosos em Atenção Domiciliar na Estratégia Saúde da Família. Kairós Gerontologia 2016;19(2):133-46.

29. Wang $\mathrm{CH}$, Tsai CL. Physical Activity Is Associated With Greater Visuospatial Cognitive Functioning Regardless of the Level of Cognitive Load in Elderly Adults. J Sport Exerc Psychol 2016;38(1):69-81. doi: 10.1123/jsep.2015-0221

30. Moraes EM; Moraes FL; Lima SPP. Características biológicas e psicológicas do envelhecimento. Revista Médica de Minas Gerais 2010;20(1):67-73.

31. Fechine BRA, Trompieri N. O processo de envelhecimento: as principais alterações que acontecem com o idoso com o passar dos anos. InterSciencePlace 2012;1(20):106-32. doi: 10.6020/1679-9844/2007

32. Freitas ER, Barbosa AJG. Qualidade de vida e bem-estar psicológico no climatério. Arq Bras Psicol 2015;67(3):112-24.

33. Fonseca $\mathrm{S}$, Amante MJ, Araújo L, Morgado M, Nunes T. O impacto de um programa de estimulação cognitiva em pessoas idosas a residir na comunidade vs. Institucionalizadas. Actas de Gerontologia 2016;2(1):1-9.

34. Rigo MLNR; Teixeira D. Efeitos da atividade física na percepção de bem-estar de idosas que residem sozinhas e acompanhadas. Journal of Health Sciences 2015;7(1):34-5. doi:10.17921/2447-8938.2005v7n1p\%25p

35. Batistoni SST. Contribuições da psicologia do envelhecimento para as práticas clínicas com idosos. Psicologia em Pesquisa 2009;3(2):13-22.

36. Antunes HKM, Santos RF, Cassilhas R, Santos RVT, Bueno OOFA, Mello MT. Exercício físico e função cognitiva: uma revisão. Rev Bras Med Esporte 2006;12(2):108-14.

37. Sposito G, Neri AL, Yassuda MS. Atividades avançadas de vida diária (AAVDs) e o desempenho cognitivo em idosos residentes na comunidade: Dados do Estudo FIBRA Polo UNICAMP. Rev Bras Geriatr Gerontol 2016;19(1):7-20. doi: 10.1590/1809-9823.2016.15044

38. Brito TRP; PavariniSCL. Relaçãoentreapoiosocialecapacidade funcional de idosos com alterações cognitivas. Revista Latino-Americana de Enfermagem 2012;20(4):677-84. doi: 10.1590/S0104-11692012000400007

39. Rabelo DF, Neri AL. Tipos de configuração familiar e condições de saúde física e psicológica em idosos. Cad Saúde Pública 2015;31(4):874-84. doi: 410.1590/0102-311X00087514
40. Minghelli B, Tomé B, Nunes C, Neves A, Simões C. Comparação dos níveis de ansiedade e depressão entre idosos ativos e sedentários. Rev Psiquiatr Clín 2013;40(2):71-6. doi: 10.1590/S0101-60832013000200004

41. Casemiro FG, Rodrigues IA, Dias JC, Alves LCS, Inouye K, Gratão ACM. Impacto da estimulação cognitiva sobre depressão, ansiedade, cognição e capacidade funcional em adultos e idosos de uma universidade aberta da terceira idade. Rev Bras Geriatr Gerontol 2016;19(4):683-94. doi: 10.1590/1809-98232016019.150214

42. Uchmanowicz I, Gobbens RJJ. The relationship between frailty, anxiety and depression, and health-related quality of life in elderly patients with heart failure. Clin Interv Aging 2015;10:1595-600. doi: 10.2147/CIA.S90077

43. Oliveira KLd, Santos AAAd, Cruvinel M, Néri AL. Relação entre ansiedade, depressão e desesperança entre grupos de idosos. Psicologia em Estudo 2006;11:351-9.

44. Delphin-Combe F, Bathsavanis A, Rouch I, Liles T, VannierNitenberg C, Fantino B, Dauphinot V, Krolak-Salmon P. Relationship between anxiety and cognitive performance in an elderly population with a cognitive complaint. Eur J Neurol 2016;23(7):1210-7. doi: 10.1111/ene.13004

45. Machado MB, Ignácio ZM, Jornada LK, Réus GZ, Abelaira HM, Arent CO, Schwalm MT, Caretta RA, Caretta LB, Quevedo J. Prevalência de transtornos ansiosos e algumas comorbidades em idosos: um estudo de base populacional. J Bras Psiquiatr 2016:65:28-35, doi: 10.1590/00472085000000100

46. Lampl C, Thomas H, Tassorelli C, Katsarava Z, Laínez JM, Lantéri-Minet M, Rastenyte D, Torre ER, Stovner LJ, Andrée C, Steiner TJ. Headache, depression and anxiety: associations in the Eurolight project. The Journal of Headache and Pain 2016;17(1):59. doi: 10.1186/s10194-016-0649-2

47. Wolitzky-Taylor KB, Castriotta N, Lenze EJ, Stanley MA, Craske MG. Anxiety disorders in older adults: a comprehensive review. Depression and anxiety 2010;27(2):190-211. doi: $10.1002 /$ da. 20653

48. Antunes HKM, Stella SG, Santos RF, Bueno OFA, Mello MTd. Depression, anxiety and quality of life scores in seniors after an endurance exercise program. Rev Bras Psiquiatr 2005;27:266-71. doi: S1516-44462005000400003

49. Paluska SA, Schwenk TL. Physical activity and mental health. Sports medicine 2000;29(3):167-80.

50. Prina AM, Ferri CP, Guerra M, Brayne C, Prince M. Prevalence of anxiety and its correlates among older adults in Latin America, India and China: cross-cultural study. Br J Psychiatry 2011;199(6):485-491. doi: 10.1192/bjp.bp.110.083915.

51. Bobevski I, Clarke DM, Meadows G. Health Anxiety and Its Relationship to Disability and Service Use: Findings From a Large Epidemiological Survey. Psychosomatic Medicine 2016;78(1):13-25. doi: 10.1097/PSY.0000000000000252

52. Cunha JA. Manual da versão em português das Escalas Beck. São Paulo: Casa do Psicólogo 2001:11-3.

53. Casemiro FG, Rodrigues IA, Dias JC, Alves LCdS, Inouye K, Gratão ACM. Impact of cognitive stimulation on depression, anxiety, cognition and functional capacity among adults and elderly participants of an open university for senior citizens. Ver Bras Geriatr Gerontol 2016;19:683-94. doi: 10.1590/1809-98232016019.150214

54. Braga MCP, Casella MA, Campos MLN, Paiva SP. Qualidade de vida medida pelo whoqol-bref: estudo com idosos residentes em Juiz de Fora/MG. Revista de Atenção Primária a Saúde 2011;14(1). 
55. Pereira MdG, Carvalho H. Qualidade vida, sobrecarga, suporte social, ajustamento conjugal e morbidade psicológica em cuidadores de idosos com dependência funcional. Temas em Psicologia 2012;20(2):369-84. doi: 10.9788/TP2012.2-07

56. Loureiro LdSN, Fernandes MdGM, Nóbrega MMLd, Rodrigues RAP. Sobrecarga em cuidadores familiares de idosos: associação com características do idoso e demanda de cuidado. Rev Bras Enfermag 2014;67:227-32. doi: 10.5935/0034-7167.20140030

57. Fazzi L, Dobrianskyj FM, Reggi JRA, Otani VHO, Otani TZS, Uchida RR. The influence of anxiety on quality of life among patients with an indication for cataract surgery.
Sao Paulo Med J 2015;133:160-1. doi: 10.1590/15163180.2014.9510109

58. Gutke A, Lundberg M, Ostgaard HC, Oberg B. Impact of postpartum lumbopelvic pain on disability, pain intensity, health-related quality of life, activity level, kinesiophobia, and depressive symptoms. Eur Spine J 2011;20(3):440-8. doi: 10.1007/s00586-010-1487-6

59. Xavier FM, Ferraz MP, Trenti CM, Argimon I, Bertollucci $\mathrm{PH}$, Poyares D, Moriguchi EH. Transtorno de ansiedade generalizada em idosos com oitenta anos ou mais. Rev Saúde Pública 2001;35:294-302. doi: 10.1590/S003489102001000300013

Como citar: VICENTINI DE OLIVEIRA, Daniel; ANTUNES, Mateus Dias; OLIVEIRA, Julimar. Ansiedade e sua relação com a qualidade de vida em idosos: revisão narrativa. Cinergis, Santa Cruz do Sul, v. 18, n. 4, out. 2017. ISSN 2177-4005. Disponível em: < https://online.unisc.br/seer/index.php/cinergis/article/view/9951 >. Acesso em: 07 dez. 2017. doi:http://dx.doi.org/10.17058/ cinergis. $18 i 4.9951$. 